\title{
THE LIMITING BEHAVIOUR OF HANSKI'S INCIDENCE FUNCTION METAPOPULATION MODEL
}

\author{
R. MCVINISH* AND \\ P. K. POLLETT, ${ }^{* *}$ University of Queensland
}

\begin{abstract}
Hanski's incidence function model is one of the most widely used metapopulation models in ecology. It models the presence/absence of a species at spatially distinct habitat patches as a discrete-time Markov chain whose transition probabilities are determined by the physical landscape. In this analysis, the limiting behaviour of the model is studied as the number of patches increases and the size of the patches decreases. Two different limiting cases are identified depending on whether or not the metapopulation is initially near extinction. Basic properties of the limiting models are derived.
\end{abstract}

Keywords: Extinction threshold; point process; stochastic patch occupancy model; SPOM; weak convergence

2010 Mathematics Subject Classification: Primary 92D40

Secondary 60J10; 60J20; 60F05

\section{Introduction}

A metapopulation is a collection of local populations occupying spatially distinct habitat patches that evolves over time due to local extinctions and colonisation events. As noted in [12], the study of metapopulations by using a stochastic patch occupancy model (SPOM) is well established in the ecology literature. A SPOM is a discrete-time Markov chain that models the presence/absence of the focal species in a network of habitat patches. One of the most widely used SPOMs is Hanski's incidence function model (IFM); see [10]. The IFM uses structural assumptions to relate the physical landscape to the transition probabilities of the Markov chain.

One drawback of using the IFM and realistic SPOMs in general is that they are difficult to analyse. Simulation methods can be employed in specific cases, but they provide no information on the behaviour of the model in general. To understand the general case, the modeller typically needs to resort to approximations. Two common classes of approximations for Markov chain models are the deterministic approximations and branching process approximations.

Ovaskainen and Hanski [24] propose a system of deterministic difference equations as an approximation to the IFM and provide a detailed analysis of this system. However, it is not clear in what sense this system of deterministic difference equations approximates the original stochastic IFM. Our analysis is perhaps closer to Ovaskainen and Cornell's [23] analysis of a related continuous-time stochastic metapopulation model on a stochastic landscape. They showed that the fraction of occupied patches is well approximated by a one-dimensional ordinary differential equation called Levins' model [16] when the dispersal range is large.

Received 12 January 2012; revision received 25 June 2013.

* Postal address: School of Mathematics and Physics, University of Queensland, Brisbane, QLD 4072, Australia.

Email address: r.mcvinish@uq.edu.au

** Email address: pkp@maths.uq.edu.au 
If the spatial aspect of the metapopulation is ignored, then the behaviour of the metapopulation model near the extinction state resembles the initial behaviour of an epidemic. A number of researchers (see, e.g. [1], [2], [3], and [4]) have used branching processes to approximate the initial spread of an epidemic in a large population. The branching process approximation is less frequently applied in the context of metapopulation modelling, but [5] provides one example.

In this paper we study the limiting behaviour of Hanski's IFM as the number of patches increases and the size of the patches decreases. The model is briefly described and the main assumptions stated in Section 2. In Section 3, we derive and study the deterministic limit. Our analysis differs from that of [23] in three ways: (i) a different scaling is used to study the limiting process, (ii) we allow the extinction probabilities to vary spatially, and (iii) we work directly with the discrete-time IFM. In Section 4, Hanski's IFM is studied when the metapopulation is initially close to extinction. Extending our earlier work [18], point process theory is used to study the limit and determine conditions under which the metapopulation has a positive probability of recovery. We conclude with a brief discussion of the results. All proofs are given in Appendix A.

\section{Model description}

The incidence function model of Hanski [10], for a metapopulation comprising $n$ patches, is a discrete-time Markov chain on $\{0,1\}^{n}$. Denote this Markov chain by $X_{t}^{n}=\left(X_{1, t}^{n}, \ldots, X_{n, t}^{n}\right)$, where $X_{i, t}^{n}=1$ if patch $i$ is occupied at time $t$ and $X_{i, t}^{n}=0$ otherwise. Patch $i$ is described by three variables; its location $z_{i}$, a weight $A_{i}>0$ which may be interpreted as the size of the patch, and $s_{i}$ which is the probability that the population occupying patch $i$ survives one time step. Let $z^{n}, A^{n}$, and $s^{n}$ denote the collections of patch variables for the entire metapopulation. We treat $z^{n}, A^{n}$, and $s^{n}$ as random variables which is reasonable if we view the landscape as the result of some random process. A similar treatment of the landscape was adopted in the metapopulation model of Ovaskainen and Cornell [23]. The transition probabilities of the Markov chain are determined by how well the patches are connected to each other and by the probability of local extinction. The connectivity measure of patch $i$ at time $t$ is defined by

$$
\tilde{C}_{i}(t)=\sum_{j \neq i} X_{j, t}^{n} D\left(z_{i}, z_{j}\right) A_{j}^{b}, \quad b>0,
$$

where $D(z, \tilde{z}) \geq 0$ is a measure of how easily a patch located at $z$ can be colonised by an individual from a patch located at $\tilde{z}$. Typically, $D(z, \tilde{z})$ is a bounded decreasing function of $\|z-\tilde{z}\|$ such as $\exp (-\alpha\|z-\tilde{z}\|), \alpha>0$. Let $f:[0, \infty) \mapsto[0,1]$ such that $f(0)=0$. Conditional on $\left(X_{t}^{n}, z^{n}, A^{n}, s^{n}\right)$, the $X_{i, t+1}^{n}(i=1, \ldots, n)$ are independent with transitions given by

$$
\mathbb{P}\left(X_{i, t+1}^{n}=1 \mid X_{t}^{n}, z^{n}, A^{n}, s^{n}\right)=s_{i} X_{i, t}^{n}+f\left(\tilde{C}_{i}(t)\right)\left(1-X_{i, t}^{n}\right) .
$$

Since the connectivity measure of patch $i$ is only important when patch $i$ is unoccupied, the transition probabilities remain unchanged if $\tilde{C}_{i}(t)$ is replaced by

$$
C_{i}(t)=\sum_{j=1}^{n} X_{j, t}^{n} D\left(z_{i,}, z_{j}\right) A_{j}^{b} .
$$

There are a couple of important differences between the IFM and typical interacting particle systems used in spatial modelling; see, e.g. [9] and [25]. Apart from the discrete time setting, 
interacting particle systems usually evolve on a lattice of sites whereas the patches of an IFM are not naturally restricted to a lattice structure. More importantly, in interacting particle systems the instantaneous effect of change at one site is assumed to be limited to a finite neighbourhood of that site. The IFM does not usually have a finite range neighbourhood. For typical choices of $D$, changes at one patch immediately affect the transition probabilities for all other patches of the metapopulation.

Our analysis of the IFM will be based on the following assumptions:

(a) $A_{i}=a_{i} n^{-1 / b}$, where $a_{i} \in(0, A]$. The collection of $a_{i}$ will be denoted by $a^{n}$;

(b) $z_{i} \in \Omega$, where $\Omega$ is a compact subset of $\mathbb{R}^{d}$;

(c) $D(z, \tilde{z})$ is symmetric in all its arguments and defines a uniformly bounded and equicontinuous family of functions on $\Omega$. That is, there exists a finite constant $\bar{D}$ such that, for all $z_{1}, z_{2} \in \Omega$, we have $\left|D\left(z_{1}, z_{2}\right)\right| \leq \bar{D}$, and, for every $\varepsilon>0$, there exists a $\delta>0$ such that, for all $z_{1}, z_{2}$ with $\left\|z_{1}-z_{2}\right\|<\delta$,

$$
\sup _{z \in \Omega}\left|D\left(z_{1}, z\right)-D\left(z_{2}, z\right)\right|<\varepsilon
$$

(d) the function $f$ is Lipschitz continuous.

Assumption (a) implies that all patches are of a comparable size. Furthermore, in applications to butterfly metapopulations, estimates of $b$ are typically less than one; see [11] and [26]. Therefore, assumption (a) corresponds to a decrease in the total area of the metapopulation as $n \rightarrow \infty$ which is consistent with habitat fragmentation and destruction. Assumption (b) is made to simplify the analysis. Assumption (c) is satisfied for the examples given in [21, Section 2.2.1]. In particular, it is satisfied for $D(z, \tilde{z})=\exp (-\alpha\|z-\tilde{z}\|), \alpha \geq 0$. Typical forms for $f$ include $f(x)=x^{2} /\left(\beta+x^{2}\right), \beta>0$, and $f(x)=1-\exp (-\beta x), \beta>0$; see [21, Section 2.2.3]. Both of these functions have bounded derivatives on $[0, \infty)$, so assumption (d) is satisfied.

Another assumption that is used in the analysis concerns the random variables $\left(z^{n}, a^{n}, s^{n}\right)$. Let $C^{+}([0,1] \times \Omega)$ denote the class of continuous functions $h:[0,1] \times \Omega \mapsto[0, \infty)$. Note that, since $\Omega$ is a compact set from assumption (b), the functions in $C^{+}([0,1] \times \Omega)$ are bounded. Consider the sequence of random measures $\sigma_{n}$ defined by

$$
\int h(s, z) \sigma_{n}(\mathrm{~d} s, \mathrm{~d} z):=n^{-1} \sum_{i=1}^{n} a_{i}^{b} h\left(s_{i}, z_{i}\right) \quad \text { for all } h \in C^{+}([0,1] \times \Omega) .
$$

The measure $\sigma_{n}$ describes the landscape of the $n$ patch metapopulation model. It is purely atomic placing mass $n^{-1} a_{i}^{b}$ at the point determined by patch $i$ 's location $z_{i}$ and its survival probability $s_{i}$. We assume that $\sigma_{n}$ satisfies the following assumption.

(e) As $n \rightarrow \infty, \sigma_{n} \stackrel{\text { D }}{\rightarrow} \sigma$ for some nonrandom measure $\sigma$.

Applying the law of large numbers and Theorem 16.16 of [15], we see that assumption (e) is satisfied if, for example, the random vectors $\left(z_{i}, a_{i}, s_{i}\right)$ are independent and identically distributed. 


\section{Deterministic limit}

Consider the array of random measures $\mu_{n, t}$ constructed from the Markov chain $X_{t}^{n}$ by

$$
\int h(s, z) \mu_{n, t}(\mathrm{~d} s, \mathrm{~d} z):=n^{-1} \sum_{i=1}^{n} a_{i}^{b} X_{i, t}^{n} h\left(s_{i}, z_{i}\right) \quad \text { for all } h \in C^{+}([0,1] \times \Omega) .
$$

The measure $\mu_{n, t}$ has a similar structure to $\sigma_{n}$; however, $\mu_{n, t}$ only involves those patches that are occupied at time $t$. These measures can be used to determine quantities such as the proportion of occupied patches weighted by the patch size. The following theorem describes the behaviour of the metapopulation as the number of patches tends to $\infty$.

Theorem 3.1. Suppose that assumptions $(a)-(e)$ hold and that $\mu_{n, 0} \stackrel{\mathrm{D}}{\rightarrow} \mu_{0}$ for some nonrandom measure $\mu_{0}$. Then $\mu_{n, t} \stackrel{\mathrm{D}}{\rightarrow} \mu_{t}$ for all $t=0,1, \ldots$, where $\mu_{t}$ is defined by the recursion

$$
\begin{aligned}
& \int h(s, z) \mu_{t+1}(\mathrm{~d} s, \mathrm{~d} z) \\
& =\int \operatorname{sh}(s, z) \mu_{t}(\mathrm{~d} s, \mathrm{~d} z)+\int h(s, z) f\left(\int D(z, \tilde{z}) \mu_{t}(\mathrm{~d} \tilde{s}, \mathrm{~d} \tilde{z})\right) \sigma(\mathrm{d} s, \mathrm{~d} z) \\
& \quad-\int h(s, z) f\left(\int D(z, \tilde{z}) \mu_{t}(\mathrm{~d} \tilde{s}, \mathrm{~d} \tilde{z})\right) \mu_{t}(\mathrm{~d} s, \mathrm{~d} z)
\end{aligned}
$$

for all $h \in C^{+}([0,1] \times \Omega)$.

Consider the special case where $D(z, \tilde{z})=1$ for all $(z, \tilde{z}) \in \Omega \times \Omega$ and we assume that $a_{i}=1$ for all $i$. Let $h(s, z)=s^{k}$ for $k=0,1,2, \ldots$ Then recursion (3.2) becomes

$$
d(t+1 ; k)=d(t ; k+1)+f(d(t ; 0))\left(\bar{s}_{k}-d(t ; k)\right),
$$

where $d(t ; k)=\int s^{k} \mu_{t}(\mathrm{~d} s, \mathrm{~d} z)$ and $\bar{s}_{k}=\int s^{k} \sigma(\mathrm{d} s, \mathrm{~d} z)$. This special case is related to the model studied in [18]. The difference between equation (3.3) and equation 2.1 of [18] is that in [18] it is assumed that extinction and colonisation events occur in distinct alternating phases.

Despite the complexity of the recursion (3.2), we are able to provide conditions for the existence of a nonzero fixed point and show that if a nonzero fixed point exists then it is unique. Fixed points are important in applications as they determine the equilibrium behaviour of the metapopulation. In particular, the presence of a nonzero fixed point implies that the metapopulation may persist for a long time.

Three further assumptions are introduced to study the fixed points of the recursion (3.2).

(f) The function $f$ is increasing, strictly concave, and twice differentiable with bounded second derivative in a neighbourhood of 0 .

(g) For some $\varepsilon>0$ sufficiently small, $\sigma([1-\varepsilon, 1] \times \Omega)=0$. Also, for every $z \in \Omega$ and every open neighbourhood $N_{z}$ of $z, \sigma\left([0,1] \times N_{z}\right)>0$.

(h) $D(z, \tilde{z})>0$ for all $(z, \tilde{z}) \in \Omega \times \Omega$.

Assumption (f) is satisfied for $f(x)=1-\exp (-\beta x), \beta>0$, but not for $f(x)=x^{2} /\left(\beta+x^{2}\right)$, $\beta>0$. In ecological terms, by introducing assumption (f) we are trying to exclude a metapopulation level Allee-like effect. An Allee-like effect refers to a metapopulation exhibiting a critical threshold in the occupancy level below which the metapopulation goes extinct; 
see [6, pp. 103-105]. Note that, even for simple models displaying an Allee-like effect, determining the number of fixed points can be a challenging problem. Assumption (g) excludes the possibility of any patches having a very large survival probability. Furthermore, for any $\mu_{n, t}$ defined by (3.1) such that $\mu_{n, t} \stackrel{\mathrm{D}}{\rightarrow} \mu_{t}$, assumption $(\mathrm{g})$ implies that $\mu_{t}([1-\varepsilon, 1] \times \Omega)=0$. Assumption (h) means that all patches are connected to each other. Although some range limited forms of $D$ have been used, the most common forms satisfy assumption (h); see [21, Section 2.2.1].

Let $\mu_{\infty}(\mathrm{d} s, \mathrm{~d} z)$ denote a fixed point of (3.2) and define $\psi(z)=\int D(z, \tilde{z}) \mu_{\infty}(\mathrm{d} \tilde{s}, \mathrm{~d} \tilde{z})$. Upon rearranging (3.2), we see that any fixed point satisfies

$$
\int h(s, z)(1-s+f(\psi(z))) \mu_{\infty}(\mathrm{d} s, \mathrm{~d} z)=\int h(s, z) f(\psi(z)) \sigma(\mathrm{d} s, \mathrm{~d} z) .
$$

By definition, $\psi(z) \geq 0$ and, by assumption (c), $\psi$ is continuous on $\Omega$. Under assumption $(\mathrm{g})$ the support of $\sigma$, and hence the support of $\mu_{\infty}$, is contained in $[0,1-\varepsilon] \times \Omega$. Therefore, for any $h \in C^{+}([0,1] \times \Omega)$, the function $h(s, z)(1-s+f(\psi(z)))^{-1}$ is a continuous function on the support of $\mu_{\infty}$. Using this fact together with equation (3.4), we see that, for any $h \in C^{+}([0,1] \times \Omega)$,

$$
\int h(s, z) \mu_{\infty}(\mathrm{d} s, \mathrm{~d} z)=\int h(s, z) \frac{f(\psi(z))}{1-s+f(\psi(z))} \sigma(\mathrm{d} s, \mathrm{~d} z) .
$$

Clearly, $\mu_{\infty}$ is absolutely continuous with respect to $\sigma$ and has Radon-Nikodym derivative

$$
\frac{\partial \mu_{\infty}}{\partial \sigma}=\frac{f(\psi(z))}{1-s+f(\psi(z))},
$$

where $\psi$ satisfies the equation

$$
\psi(z)=\int D(z, \tilde{z}) \frac{f(\psi(\tilde{z}))}{1-\tilde{s}+f(\psi(\tilde{z}))} \sigma(\mathrm{d} \tilde{s}, \mathrm{~d} \tilde{z}) .
$$

The number of solutions to equation (3.5) is determined by the following theorem.

Theorem 3.2. Suppose that assumptions $(b)-(d)$ and $(f)-(h)$ hold. Let $\mathcal{A}: C(\Omega) \mapsto C(\Omega)$ be the bounded linear operator

$$
\mathcal{A} \phi(z)=f^{\prime}(0) \int \frac{D(z, \tilde{z})}{(1-\tilde{s})} \phi(\tilde{z}) \sigma(\mathrm{d} \tilde{s}, \mathrm{~d} \tilde{z}), \quad \phi \in C(\Omega),
$$

and let $r(\mathcal{A})$ be the spectral radius of $\mathcal{A}$. If $r(\mathcal{A}) \leq 1$ then $\psi=0$ is the unique solution to equation (3.5). If $r(\mathcal{A})>1$ then (3.5) has two solutions of which one is $\psi=0$.

Theorem 3.2 simplifies considerably for certain special cases. For example, consider the case where $D(z, \tilde{z})=\exp \left(-\theta\left(\left\|z-z_{0}\right\|+\left\|\tilde{z}-z_{0}\right\|\right)\right)$ for some $\theta>0$ and $z_{0} \in \Omega$. This form for $D(z, \tilde{z})$ can be interpreted as follows: individuals moving from a patch located at $\tilde{z}$ to a patch located at $z$ are required to first go through the point $z_{0}$. To apply Theorem 3.2, we note that $\mathcal{A}$ maps continuous functions to functions of the form $C \exp \left(-\theta\left\|z-z_{0}\right\|\right)$ for some $C>0$. Therefore, the spectral radius of $\mathcal{A}$ is easily computed to be

$$
f^{\prime}(0) \int \frac{\exp \left(-2 \theta\left\|\tilde{z}-z_{0}\right\|\right)}{1-\tilde{s}} \sigma(\mathrm{d} \tilde{s}, \mathrm{~d} \tilde{z}) .
$$

Further discussion of metapopulations with $D$ of this form is given in [20]. 
To end this section we comment on the problem of stability of the fixed points. Although we have been unable to determine the stability of these fixed points, we conjecture that if $r(\mathcal{A})>1$ then the nonzero fixed point is asymptotically stable and the zero fixed point is unstable. Otherwise, if $r(\mathcal{A}) \leq 1$ then the zero fixed point is asymptotically stable. In other words, we conjecture that this metapopulation will persist for a long time if $r(\mathcal{A})>1$ but will go extinct quickly if $r(\mathcal{A}) \leq 1$, a property called the extinction threshold. In support of this conjecture, note that if the extinction and colonisation events were assumed to occur in distinct alternating phases, as in [18] and [20], then stability of the fixed points could be established using similar arguments to those used to prove Theorem 3 of [20].

\section{Point process limit}

We now consider the case where initially only a small number of habitat patches are occupied. Although we could still apply Theorem 3.1, we would not obtain any useful information about the metapopulation. In that case, $\mu_{n, 0}$ converges to the trivial measure which implies that $\mu_{t}$ is the trivial measure for all $t \geq 0$. A different analysis is needed to understand the behaviour of the metapopulation model near the extinction state. We replace assumption (a) by

(a') $A_{i}=n^{-1 / b}$ for all $i$.

Assumption $\left(\mathrm{a}^{\prime}\right)$ implies that all habitat patches are of the same size. Note that, from assumption (c), $D(z, \tilde{z})$ can be continuously extended to all $\mathbb{R}^{d}$ so that $D(\cdot, \tilde{z})=0$ for all $\tilde{z} \notin \Omega^{\prime}$, where $\Omega^{\prime}$ is a compact subset of $\mathbb{R}^{d}$ and $\Omega \subset \Omega^{\prime}$.

As in [18], we define the point processes associated with the IFM using a random counting measure. Let $\mu_{n, t}$ denote the random measure defined by

$$
\mu_{n, t}(B):=\#\left\{\left(s_{i}, z_{i}\right) \in B: X_{i, t}^{n}=1\right\}
$$

for any bounded Borel set $B$. Note that the definition of $\mu_{n, t}$ given at (4.1) has two important differences to the one given at (3.1); all patches are of the same size and there is no longer a scaling by $n^{-1}$. The main tool that we shall use in the analysis of these point processes is the probability generating functional. Let $\mathcal{V}$ denote the class of all real-valued Borel functions $h$ on $\mathbb{R}^{d+1}$ with $1-h$ vanishing outside some bounded set and satisfying $0 \leq h(s, z) \leq 1$ for all $(s, z) \in \mathbb{R}^{d+1}$. The probability generating functional of the point process $\mu_{n, t}$ is defined by

$$
G_{n, t}[h]:=\mathbb{E}\left(\exp \left(\int \log h(s, z) \mu_{n, t}(\mathrm{~d} s, \mathrm{~d} z)\right)\right)=\mathbb{E}\left(\prod_{i=1}^{n}\left(X_{i, t}^{n} h\left(s_{i}, z_{i}\right)+1-X_{i, t}^{n}\right)\right) .
$$

Moyal [22] introduced a generalisation of branching processes called multiplicative population chains. Multiplicative population chains (MPC) are a family of point processes that evolve in discrete time such that the point process at time $t+1$ is a superposition of conditionally independent point processes representing the offspring from each element of the point process at time $t$. Let $G_{t}[\cdot \mid(s, z)]$ denote the probability generating functional of the MPC at time $t$ given that the MPC at time 0 consisted of a single element located at $(s, z)$. Moyal [22, Theorem 1.1] showed that the probability generating functional of an MPC satisfies the functional relation

$$
G_{t}[h \mid(s, z)]=G_{u}\left[G_{t-u}[h \mid \cdot] \mid(s, z)\right], \quad u=1, \ldots, t-1 .
$$

Given the probability generating functional at time 0 , denoted by $G_{0}$, the probability generating functional at time $t$ can be obtained from the recursion (4.2) by $G_{t}[h]=G_{0}\left[G_{t}[h \mid(s, z)]\right]$. In the following theorem we show that, under certain assumptions, the IFM converges to an MPC and give the recursion for the probability generating functional. 
Theorem 4.1. Suppose that assumptions $\left(a^{\prime}\right)$ and $(b)-(f)$ hold. Assume that the sequence of point processes $\left\{\mu_{n, 0}\right\}_{n=1}^{\infty}$ converges weakly to some point process with probability generating functional $G_{0}$ and that $\sup _{n} \mathbb{E}\left(\exp \left(\alpha \sum_{i=1}^{n} X_{i, 0}^{n}\right)\right)<\infty$ for all $\alpha>0$. The sequence of point processes $\left\{\mu_{n, t}\right\}_{n=1}^{\infty}$ converges weakly to the point process with probability generating functional given by the recursion

$$
G_{t+1}[h]=G_{t}\left[G_{1}[h \mid(s, z)]\right] \text { for any } h \in \mathcal{V},
$$

where

$$
G_{1}[h \mid(s, z)]=(1-U(s)(1-h(s, z))) \exp \left(-f^{\prime}(0) \int D(\tilde{z}, z)(1-h(\tilde{s}, \tilde{z})) \sigma(\mathrm{d} \tilde{s}, \mathrm{~d} \tilde{z})\right)
$$

and $U$ is the distribution function of the standard uniform distribution.

As is the case with branching process approximations of Markov chains, of particular interest is the probability that the limiting MPC goes extinct in finite time. Moyal [22, Theorem 3.1] shows that to determine the extinction probability we need to find the smallest nonnegative solution to the functional equation $h=G_{1}[h \mid(s, z)], h \in \mathcal{V}$. Clearly, one solution is given by $h(s, z)=1$ for all $(s, z) \in \mathbb{R}^{d+1}$. Therefore, we need to determine conditions under which smaller solutions exist.

Theorem 4.2. Suppose that assumptions $(b),(c),(g)$, and $(h)$ hold. The probability that the limiting MPC goes extinct in finite time is less than one if and only if $r(\mathcal{A})>1$, where $\mathcal{A}$ is the bounded linear operator defined by equation (3.6). If $r(\mathcal{A})>1$ then the probability of extinction in finite time is given by

$$
G_{0}\left[\frac{(1-U(s)) \psi^{*}(z)}{1-U(s) \psi^{*}(z)}\right],
$$

where $\psi^{*}$ is the smallest nonnegative solution to

$$
\psi(z)=\exp \left(-f^{\prime}(0) \int D(\tilde{z}, z)\left(\frac{1-\psi(\tilde{z})}{1-U(\tilde{s}) \psi(\tilde{z})}\right) \sigma(\mathrm{d} \tilde{s}, \mathrm{~d} \tilde{z})\right) \text { for all } z \in \Omega .
$$

When $D(z, \tilde{z})=1$ for all $(z, \tilde{z}) \in \Omega \times \Omega$, the results of this section are parallel to those of [18, Section 3] with the small differences being due to the phase structure of colonisation and extinction events assumed in [18]. The proof of Theorem 4.2 (Appendix A.4) uses similar functional analytic tools to those used in the proof of Theorem 3.2 (Appendix A.2).

Theorem 4.2 provides further support to our conjecture concerning the stability of the fixed points of (3.2). Suppose that $r(\mathcal{A})>1$, then we conjectured that 0 is an unstable fixed point of the recursion (3.2). If this were not the case then the metapopulation would display very strange behaviour. Theorem 4.2 indicates that if the initial number of occupied patches is very small compared to the number of patches in the metapopulation and $r(\mathcal{A})>1$ then the number of occupied patches will increase and the metapopulation will persist for a long time with positive probability. However, if 0 were a stable fixed point of recursion (3.2) and a small but positive proportion of patches are initially occupied in the metapopulation then there should be a rapid decrease to 0 in the proportion of occupied patches.

\section{Discussion}

In this paper, we studied the convergence of the IFM under two different sets of initial conditions. One of the goals of studying convergence was to derive an approximation of the IFM which would allow a simpler analysis. Although both of the derived limiting processes 
are still quite complex, we have been able to establish some of their features. One benefit of determining the limiting processes is that the effect of the model parameters is more transparent. For example, it can be demonstrated that those factors that we would expect to improve the likelihood of the metapopulation persisting, such as a more rapidly increasing colonisation function and easier movement between patches, lead to an increase in the nonzero equilibrium of the deterministic limit from Theorem 3.1 and a decrease in the probability of extinction in the MPC limit from Theorem 4.1.

Determining the stability of the fixed points of (3.2) remains a significant challenge. We have conjectured that the nonzero fixed point of (3.2), when it exists, is stable and that when only the zero fixed point exists, the zero fixed point is stable. A resolution of this problem is necessary before more complex dynamics, such as a metapopulation Allee-like effect, can be incorporated.

\section{Appendix A. Proofs}

\section{A.1. Proof of Theorem 3.1}

We essentially proceed as in the proof of Theorem 2.1 of [18]. First note that if $\int h \mathrm{~d} \mu_{n} \stackrel{\mathrm{D}}{\rightarrow}$ $\int h \mathrm{~d} \mu$ for all $h \in C^{+}([0,1] \times \Omega)$ then $\mu_{n} \stackrel{\mathrm{D}}{\rightarrow} \mu$; see [15, Theorem 16.16]. We use induction on $t$ to prove weak convergence of the random measures $\mu_{n, t}$ to the nonrandom measures $\mu_{t}$. By assumption $\mu_{n, 0} \stackrel{\mathrm{D}}{\rightarrow} \mu_{0}$ for some nonrandom measure $\mu_{0}$. Suppose that $\mu_{n, t} \stackrel{\mathrm{D}}{\rightarrow} \mu_{t}$ for some nonrandom measure $\mu_{t}$. Then

$$
\begin{aligned}
\mathbb{E}\left(\int\right. & \left.h \mathrm{~d} \mu_{n, t+1} \mid X_{t}^{n}, s^{n}, a^{n}, z^{n}\right) \\
= & n^{-1} \sum_{i=1}^{n} a_{i}^{b} h\left(s_{i}, z_{i}\right) \mathbb{E}\left(X_{i, t+1}^{n} \mid X_{t}^{n}, s^{n}, a^{n}, z^{n}\right) \\
= & n^{-1} \sum_{i=1}^{n} a_{i}^{b} s_{i} h\left(s_{i}, z_{i}\right) X_{i, t}^{n} \\
& +n^{-1} \sum_{i=1}^{n} a_{i}^{b} h\left(s_{i}, z_{i}\right) f\left(n^{-1} \sum_{j=1}^{n} a_{j}^{b} D\left(z_{i}, z_{j}\right) X_{j, t}^{n}\right)\left(1-X_{i, t}^{n}\right) \\
= & \int s h(s, z) \mu_{n, t}(\mathrm{~d} s, \mathrm{~d} z)+\int h(s, z) f\left(\int D(z, \tilde{z}) \mu_{n, t}(\mathrm{~d} \tilde{s}, \mathrm{~d} \tilde{z})\right) \sigma_{n}(\mathrm{~d} s, \mathrm{~d} z) \\
& -\int h(s, z) f\left(\int D(z, \tilde{z}) \mu_{n, t}(\mathrm{~d} \tilde{s}, \mathrm{~d} \tilde{z})\right) \mu_{n, t}(\mathrm{~d} s, \mathrm{~d} z) \\
= & \int s h(s, z) \mu_{n, t}(\mathrm{~d} s, \mathrm{~d} z)+\int h(s, z) f\left(\int D(z, \tilde{z}) \mu_{t}(\mathrm{~d} \tilde{s}, \mathrm{~d} \tilde{z})\right) \sigma_{n}(\mathrm{~d} s, \mathrm{~d} z) \\
& -\int h(s, z) f\left(\int D(z, \tilde{z}) \mu_{t}(\mathrm{~d} \tilde{s}, \mathrm{~d} \tilde{z})\right) \mu_{n, t}(\mathrm{~d} s, \mathrm{~d} z)+\varepsilon_{n, t}(h),
\end{aligned}
$$

where

$$
\begin{aligned}
\varepsilon_{n, t}(h)= & \int h(s, z)\left\{f\left(\int D(z, \tilde{z}) \mu_{n, t}(\mathrm{~d} \tilde{s}, \mathrm{~d} \tilde{z})\right)-f\left(\int D(z, \tilde{z}) \mu_{t}(\mathrm{~d} \tilde{s}, \mathrm{~d} \tilde{z})\right)\right\} \sigma_{n}(\mathrm{~d} s, \mathrm{~d} z) \\
& -\int h(s, z)\left\{f\left(\int D(z, \tilde{z}) \mu_{n, t}(\mathrm{~d} \tilde{s}, \mathrm{~d} \tilde{z})\right)-f\left(\int D(z, \tilde{z}) \mu_{t}(\mathrm{~d} \tilde{s}, \mathrm{~d} \tilde{z})\right)\right\} \mu_{n, t}(\mathrm{~d} s, \mathrm{~d} z) .
\end{aligned}
$$


By assumption (d) $f$ is Lipschitz continuous, so there exists an $L>0$ such that

$$
\begin{aligned}
&\left|\varepsilon_{n, t}(h)\right| \leq L \int h(s, z)\left|\int D(z, \tilde{z}) \mu_{n, t}(\mathrm{~d} \tilde{s}, \mathrm{~d} \tilde{z})-\int D(z, \tilde{z}) \mu_{t}(\mathrm{~d} \tilde{s}, \mathrm{~d} \tilde{z})\right| \\
& \times\left(\sigma_{n}(\mathrm{~d} s, \mathrm{~d} z)+\mu_{n, t}(\mathrm{~d} s, \mathrm{~d} z)\right) \\
& \leq 2 L\left(\int h(s, z) \sigma_{n}(\mathrm{~d} s, \mathrm{~d} z)\right) \sup _{z \in \Omega}\left|\int D(z, \tilde{z}) \mu_{n, t}(\mathrm{~d} \tilde{s}, \mathrm{~d} \tilde{z})-\int D(z, \tilde{z}) \mu_{t}(\mathrm{~d} \tilde{s}, \mathrm{~d} \tilde{z})\right| .
\end{aligned}
$$

From a small modification of Theorem 3.1 of [27] and assumption (c), it follows that if $\mu_{n, t} \stackrel{\mathrm{D}}{\rightarrow} \mu_{t}$, where $\mu_{t}$ is a nonrandom measure, then

$$
\sup _{z \in \Omega}\left|\int D(z, \tilde{z}) \mu_{n, t}(\mathrm{~d} \tilde{s}, \mathrm{~d} \tilde{z})-\int D(z, \tilde{z}) \mu_{t}(\mathrm{~d} \tilde{s}, \mathrm{~d} \tilde{z})\right| \stackrel{\mathbb{P}}{\rightarrow} 0 .
$$

Since $h \in C^{+}([0,1] \times \Omega)$, both $\operatorname{sh}(s, z)$ and $h(s, z) f\left(\int D(z, \tilde{z}) \mu_{t}(\mathrm{~d} \tilde{s}, \mathrm{~d} \tilde{z})\right)$ are elements of $C^{+}([0,1] \times \Omega)$, the latter being a consequence of assumption (c). This implies that

$$
\begin{aligned}
\mathbb{E}\left(\int h \mathrm{~d} \mu_{n, t+1} \mid X_{t}^{n}, s^{n}, a^{n}, z^{n}\right) \stackrel{\mathbb{P}}{\rightarrow} & \int s h(s, z) \mu_{t}(\mathrm{~d} s, \mathrm{~d} z) \\
& +\int h(s, z) f\left(\int D(z, \tilde{z}) \mu_{t}(\mathrm{~d} \tilde{s}, \mathrm{~d} \tilde{z})\right) \sigma(\mathrm{d} s, \mathrm{~d} z) \\
& -\int h(s, z) f\left(\int D(z, \tilde{z}) \mu_{t}(\mathrm{~d} \tilde{s}, \mathrm{~d} \tilde{z})\right) \mu_{t}(\mathrm{~d} s, \mathrm{~d} z) .
\end{aligned}
$$

Now to compute the conditional variance of $\int h(s, z) \mu_{n, t+1}(\mathrm{~d} s, \mathrm{~d} z)$ given $X_{t}^{n}$ :

$$
\begin{aligned}
\operatorname{var}\left(\int h \mathrm{~d} \mu_{n, t+1} \mid X_{t}^{n}, s^{n}, a^{n}, z^{n}\right) \\
\quad=\operatorname{var}\left(n^{-1} \sum_{i=1}^{n} a_{i}^{b} X_{i, t+1}^{n} h\left(s_{i}, z_{i}\right) \mid X_{t}^{n}, s^{n}, a^{n}, z^{n}\right) \\
\quad=n^{-2} \sum_{i=1}^{n} a_{i}^{2 b} h^{2}\left(s_{i}, z_{i}\right)\left(s_{i}\left(1-s_{i}\right) X_{i, t}^{n}+f\left(C_{i}(t)\right)\left(1-f\left(C_{i}(t)\right)\right)\left(1-X_{i, t}^{n}\right)\right) \\
\quad \leq n^{-2} \sum_{i=1}^{n} a_{i}^{2 b} h^{2}\left(s_{i}, z_{i}\right) .
\end{aligned}
$$

From assumptions (a) and (b), the conditional variance goes to 0 in probability. Applying a Chebyshev type inequality [19, Appendix C], we see that $\int h \mathrm{~d} \mu_{n, t+1}$ converges to $\int h \mathrm{~d} \mu_{t+1}$ in probability which is equivalent to convergence in distribution since $\int h \mathrm{~d} \mu_{t+1}$ is nonrandom.

\section{A.2. Proof of Theorem 3.2}

The proof of Theorem 3.2 depends on the theory concerning positive operators and monotone operators. We recall some definitions and notation following [8, Chapter VIII, Appendix].

Let $C(\Omega)$ be the Banach space of continuous functions on $\Omega$ (recall $\Omega$ is compact by assumption (b)). Let $K$ denote the reproducing cone of functions $h$ on $\Omega$ such that $h(z) \geq 0$ for all $z \in \Omega$ and let $\stackrel{\circ}{K}$ denote the interior of $K$. For any $\phi, \chi \in K$ we write $\phi \leq \chi$ if $\chi-\phi \in K$. 
We have previously noted that the function $\psi$ defined by $\psi(z)=\int D(z, \tilde{z}) \mu_{\infty}(\mathrm{d} \tilde{s}, \mathrm{~d} \tilde{z})$ is in $K$. Let $R: K \mapsto K$ be the operator defined by the right-hand side of (3.5). The following properties of $R$ will be used to prove Theorem 3.2.

Proposition A.1. Suppose that assumptions $(b)-(d)$ and $(f)-(h)$ hold. The operator $R$ has the following properties:

(i) continuity;

(ii) order compactness, that is, for any $\chi_{1}, \chi_{2} \in K, R$ maps the set $\left\{\phi: \phi \leq \chi_{2}, \chi_{1} \leq \phi\right\}$ to a relatively compact set;

(iii) monotonicity, that is, if $\phi_{1} \leq \phi_{2}$ then $R \phi_{1} \leq R \phi_{2}$;

(iv) strong positivity, that is, if $\phi \in K \backslash\{0\}$ then $R \phi \in \stackrel{\circ}{K}$;

(v) strong sublinearity, that is, if $\lambda \in(0,1)$ and $\phi \in \stackrel{\circ}{K}$ then $R(\lambda \phi)-\lambda R \phi \in \stackrel{\circ}{K}$.

Proof. (i) Continuity. We wish to show that for any $\varepsilon>0$ there exists a $\delta$ such that for any $\phi_{1}, \phi_{2} \in K$,

$$
\sup _{z \in \Omega}\left|\phi_{1}(z)-\phi_{2}(z)\right| \leq \delta \quad \text { implies that } \sup _{z \in \Omega}\left|R \phi_{1}(z)-R \phi_{2}(z)\right| \leq \varepsilon .
$$

Now

$$
\begin{aligned}
\left|R \phi_{1}(z)-R \phi_{2}(z)\right| & =\left|\int D(z, \tilde{z})\left[\frac{f\left(\phi_{1}(\tilde{z})\right)}{1-\tilde{s}+f\left(\phi_{1}(\tilde{z})\right)}-\frac{f\left(\phi_{2}(\tilde{z})\right)}{1-\tilde{s}+f\left(\phi_{2}(\tilde{z})\right)}\right] \sigma(\mathrm{d} \tilde{s}, \mathrm{~d} \tilde{z})\right| \\
& \leq \int D(z, \tilde{z}) \frac{(1-\tilde{s})\left|f\left(\phi_{1}(\tilde{z})\right)-f\left(\phi_{2}(\tilde{z})\right)\right|}{\left(1-\tilde{s}+f\left(\phi_{1}(\tilde{z})\right)\right)\left(1-\tilde{s}+f\left(\phi_{2}(\tilde{z})\right)\right)} \sigma(\mathrm{d} \tilde{s}, \mathrm{~d} \tilde{z}) \\
& \leq L \int D(z, \tilde{z})(1-\tilde{s})^{-1}\left|\phi_{1}(\tilde{z})-\phi_{2}(\tilde{z})\right| \sigma(\mathrm{d} \tilde{s}, \mathrm{~d} \tilde{z})
\end{aligned}
$$

since $f$ is Lipschitz continuous from assumption (d). If $\sup _{z \in \Omega}\left|\phi_{1}(z)-\phi_{2}(z)\right| \leq \delta$, then $\left|R \phi_{1}(z)-R \phi_{2}(z)\right| \leq L \delta \bar{D} \int(1-\tilde{s})^{-1} \sigma(\mathrm{d} \tilde{s}, \mathrm{~d} \tilde{z})$ by assumption (c). By assumption $(\mathrm{g})$, this integral is finite. By setting $\delta=\varepsilon\left(L \bar{D} \int(1-\tilde{s})^{-1} \sigma(\mathrm{d} \tilde{s}, \mathrm{~d} \tilde{z})\right)^{-1}$, the continuity of $R$ is established.

(ii) Order compactness. A subset $Y$ of a metric space $X$ is relatively compact if any bounded sequence in $Y$ has a subsequence which converges in $X$. To prove relative compactness we use the Arzelà-Ascoli theorem [15, Theorem A2.1] which, in this context, says that any sequence of functions $\phi_{1}, \phi_{2}, \ldots$ in $C(\Omega)$ that is uniformly bounded and equicontinuous has a subsequence that converges in $C(\Omega)$.

Take any $\chi_{1}, \chi_{2} \in K$. Let $\phi_{1}, \phi_{2}, \ldots$ be a sequence of functions in $K$ such that $\chi_{1} \leq \phi_{i} \leq \chi_{2}$ for all $i$. It is sufficient to show that the sequence of functions $R \phi_{1}, R \phi_{2}, \ldots$ is uniformly bounded and equicontinuous. For any $\phi \in K$ and all $z \in \Omega$,

$$
R \phi(z) \leq \int D(z, \tilde{z}) \sigma(\mathrm{d} \tilde{s}, \mathrm{~d} \tilde{z}) \leq \bar{D} \int \sigma(\mathrm{d} \tilde{s}, \mathrm{~d} \tilde{z})
$$

from assumption (c). Hence, the sequence $R \phi_{1}, R \phi_{2}, \ldots$ is uniformly bounded. To prove that 
the sequence is equicontinuous, take $z_{1}, z_{2} \in \Omega$ and $\phi \in K$. Then

$$
\begin{aligned}
\left|R \phi\left(z_{1}\right)-R \phi\left(z_{2}\right)\right| & \leq\left|\int\left(D\left(z_{1}, \tilde{z}\right)-D\left(z_{2}, \tilde{z}\right)\right) \frac{f(\phi(\tilde{z}))}{1-\tilde{s}+f(\phi(\tilde{z}))} \sigma(\mathrm{d} \tilde{s}, \mathrm{~d} \tilde{z})\right| \\
& \leq \int\left|D\left(z_{1}, \tilde{z}\right)-D\left(z_{2}, \tilde{z}\right)\right| \sigma(\mathrm{d} \tilde{s}, \mathrm{~d} \tilde{z}) .
\end{aligned}
$$

It now follows from assumption (c) that the sequence of functions $R \phi_{1}, R \phi_{2}, \ldots$ is equicontinuous. Hence, $R$ is order compact.

(iii) Monotonicity. For a given $s \in[0,1-\varepsilon]$, define the function

$$
g_{s}(x)=f(x)(1-s+f(x))^{-1}, \quad x \in[0, \infty) .
$$

From assumption (f), $f(x)$ is increasing, hence, $g_{s}(x)$ is increasing. It follows that if $\phi_{1} \leq \phi_{2}$ then

$$
\int D(z, \tilde{z}) \frac{f\left(\phi_{1}(\tilde{z})\right)}{1-\tilde{s}+f\left(\phi_{1}(\tilde{z})\right)} \sigma(\mathrm{d} \tilde{s}, \mathrm{~d} \tilde{z}) \leq \int D(z, \tilde{z}) \frac{f\left(\phi_{2}(\tilde{z})\right)}{1-\tilde{s}+f\left(\phi_{2}(\tilde{z})\right)} \sigma(\mathrm{d} \tilde{s}, \mathrm{~d} \tilde{z})
$$

for all $z \in \Omega$. Hence, $R \phi_{1} \leq R \phi_{2}$.

(iv) Strong positivity. Since $\phi \in K \backslash\{0\}$, there exists a $z_{0} \in \Omega$ and $\delta>0$ such that $\phi(z)>\delta$ for all $z$ in an open neighbourhood $N_{0}$ of $z_{0}$. Therefore,

$$
\begin{aligned}
\inf _{z \in \Omega} R \phi(z) & \geq \inf _{z \in \Omega} \int_{[0,1] \times N_{0}} D(z, \tilde{z}) \frac{f(\phi(\tilde{z}))}{1-\tilde{s}+f(\phi(\tilde{z}))} \sigma(\mathrm{d} \tilde{s}, \mathrm{~d} \tilde{z}) \\
& \geq\left(\inf _{z \in \Omega} \inf _{\tilde{z} \in N_{0}} D(z, \tilde{z})\right) \frac{f(\delta)}{1+f(\delta)} \sigma\left([0,1] \times N_{0}\right),
\end{aligned}
$$

which is positive from assumptions (g) and (h). Hence, $R \phi \in \stackrel{\circ}{K}$ for any $\phi \in K \backslash\{0\}$.

(v) Strong sublinearity. For any $\lambda \in(0,1)$ and $\phi \in \stackrel{\circ}{K}$,

$$
\begin{aligned}
R(\lambda \phi)-\lambda R \phi= & \int D(z, \tilde{z})\left[\frac{f(\lambda \phi(\tilde{z}))}{1-\tilde{s}+f(\lambda \psi(\tilde{z}))}-\frac{\lambda f(\lambda \phi(\tilde{z}))}{1-\tilde{s}+f(\psi(\tilde{z}))}\right] \sigma(\mathrm{d} \tilde{s}, \mathrm{~d} \tilde{z}) \\
= & \int D(z, \tilde{z}) \frac{f(\lambda \phi(\tilde{z}))-\lambda f(\phi(\tilde{z}))}{1-\tilde{s}+f(\lambda \psi(\tilde{z}))} \sigma(\mathrm{d} \tilde{s}, \mathrm{~d} \tilde{z}) \\
& +\int D(z, \tilde{z})\left[\frac{\lambda f(\phi(\tilde{z}))}{1-\tilde{s}+f(\lambda \psi(\tilde{z}))}-\frac{\lambda f(\phi(\tilde{z}))}{1-\tilde{s}+f(\psi(\tilde{z}))}\right] \sigma(\mathrm{d} \tilde{s}, \mathrm{~d} \tilde{z}) \\
\geq & \int D(z, \tilde{z}) \frac{f(\lambda \phi(\tilde{z}))-\lambda f(\phi(\tilde{z}))}{1-\tilde{s}+f(\lambda \psi(\tilde{z}))} \sigma(\mathrm{d} \tilde{s}, \mathrm{~d} \tilde{z}) .
\end{aligned}
$$

From assumption (f), $f$ is strictly concave giving $f(\lambda x)-\lambda f(x)>0$ for any $x>0$. Arguing as in the proof of part (iv), we see that this last integral is positive for all $z \in \Omega$ and this completes the proof of Proposition A.1.

Let $R^{n} \phi$ denote the $n$th application of the operator $R$ to $\phi$. From Proposition A.1, we can apply the cone limit trichotomy (see [13, Theorem 6.3]) to show that one of the following holds:

- for any $\phi \in K \backslash\{0\}$, the sequence $\left\{R^{n} \phi\right\}_{n=0}^{\infty}$ is unbounded;

- for any $\phi \in K$, the sequence $\left\{R^{n} \phi\right\}_{n=0}^{\infty}$ converges to 0 which is the unique fixed point of $R$; 
- for any $\phi \in K \backslash\{0\}$, the sequence $\left\{R^{n} \phi\right\}_{n=0}^{\infty}$ converges to $\psi$ which is the unique nonzero fixed point of $R$.

From equation (A.1) we may exclude the first possibility. It remains to determine conditions that will decide which of the latter two possibilities hold. For this, we briefly summarise some theory from functional analysis which extends the Perron-Frobenius theorem.

Let $\Lambda: C(\Omega) \mapsto \mathbb{R}$ denote a linear functional on $C(\Omega)$. A positive linear functional is a linear functional with the property that $\Lambda g \geq 0$ if $g \geq 0$ on $\Omega$. Since $\Omega$ is compact, the Riesz-Markov theorem (see [17, Section A.5]) states that a positive linear functional on $C(\Omega)$ can be expressed as

$$
\Lambda(g)=\int g \mathrm{~d} m^{*} \text { for all } g \in C(\Omega),
$$

for a unique positive Borel measure $m^{*}$ on $\Omega$. The set of positive Borel measures on $\Omega$ forms the dual cone $K^{*}$. Let $\stackrel{\circ}{K}^{*}$ denote the interior of $K^{*}$. If the unique Borel measure $m^{*}$ is identified for a positive linear functional then we write the linear functional as $\left\langle g, m^{*}\right\rangle:=\Lambda(g)$.

Theorem A.1. (Krein-Rutman theorem.) Let $K$ be a reproducing cone, with interior $\stackrel{\circ}{K} \neq \varnothing$, and let $B$ be a strongly positive compact bounded linear operator on $K$. Then the spectral radius of $B, r(B)$, is a simple eigenvalue of $B$ and $B^{*}$ (the adjoint of $\left.B\right)$, and their associated eigenvectors belong to $\stackrel{\circ}{K}^{\text {and } K^{*}}$. Furthermore, all other eigenvalues are strictly less in absolute value than $r(B)$.

Corollary A.1. Let $B$ be a strongly positive compact bounded linear operator, $s$ a positive number, and $\phi \in K \backslash\{0\}$ satisfying $s \phi \leq B \phi$. Then $s \leq r(B)$ with equality if and only if $s \phi=B \phi$.

Proof. Let $u^{*}$ be the eigenvector of $B^{*}$ associated with the eigenvalue $r(B)$. From Theorem A. $1, u^{*} \in \stackrel{\circ}{K}^{*}$. Since $B \phi-s \phi \in K$, we have $0 \leq\left\langle B \phi-s \phi, u^{*}\right\rangle$ with equality if and only if $s \phi=B \phi$. Now

$$
\left\langle B \phi-s \phi, u^{*}\right\rangle=\left\langle B \phi, u^{*}\right\rangle-s\left\langle\phi, u^{*}\right\rangle=\left\langle\phi, B^{*} u^{*}\right\rangle-s\left\langle\phi, u^{*}\right\rangle=(r(B)-s)\left\langle\phi, u^{*}\right\rangle .
$$

Since $u^{*} \in \stackrel{\circ}{K}^{*}$ and $\phi \neq 0$ we have $\left\langle\phi, u^{*}\right\rangle>0$. Hence, $s \leq r(B)$ with $s=r(B)$ if and only if $B \phi=s \phi$. This completes the proof of Corollary A.1.

Adapting the arguments of Proposition A.1, we see that, under assumptions (c), (g), and (h), $\mathcal{A}$ is a strongly positive compact operator. Therefore, the Krein-Rutman theorem can be applied to $\mathcal{A}$. The next proposition shows that the operator $R$ can be bounded by $\mathcal{A}$.

Proposition A.2. For any $\phi \in K, R \phi \leq \mathcal{A} \phi$ with equality if and only if $\phi=0$.

Proof. From assumption (f), $f$ is strictly concave and, therefore, the function $g$, defined in (A.2), is also strictly concave in $x$. For any $x>0$ and $s \in[0,1)$,

$$
\frac{f(x)}{1-s+f(x)}<\frac{f^{\prime}(0)}{1-s} x
$$

For any $\phi \in K \backslash\{0\}$, the set $\{(s, z): 0<\phi(z)\}$ will have positive $\sigma$ measure by assumption $(\mathrm{g})$. Therefore, for all $\phi \in K$ and all $z \in \Omega$,

$$
\int D(z, \tilde{z}) \frac{f(\phi(\tilde{z}))}{1-\tilde{s}+f(\phi(\tilde{z}))} \sigma(\mathrm{d} \tilde{s}, \mathrm{~d} \tilde{z})<\int D(z, \tilde{z}) \frac{f^{\prime}(0)}{(1-\tilde{s})} \phi(\tilde{z}) \sigma(\mathrm{d} \tilde{s}, \mathrm{~d} \tilde{z}),
$$


by assumption (h). In other words, the inequality $R \phi \leq \mathcal{A} \phi$ is strict for any $\phi \in K \backslash\{0\}$. To complete the proof, we simply note that if $\phi=0$ then $R \phi=\mathcal{A} \phi$.

We are now able to prove the first part of Theorem 3.2.

Lemma A.1. If $r(\mathcal{A}) \leq 1$ then $\psi=0$ is the unique solution to equation (3.5).

Proof. Suppose that there exists a $\phi \in K \backslash\{0\}$ such that $\phi=R \phi$. From Proposition A.2, $\phi \leq \mathcal{A} \phi$. From Corollary A.1, this is only possible if $r(\mathcal{A}) \geq 1$. So, if $r(\mathcal{A})<1$ then the only solution to equation (3.5) is $\psi=0$. Now suppose that $r(\mathcal{A})=1$. Then Corollary A.1 implies that $\phi=\mathcal{A} \phi$. From Proposition A.2, this is only possible if $\phi=0$.

It remains to consider the case where $r(\mathcal{A})>1$.

Lemma A.2. If $r(\mathcal{A})>1$ then there exists a unique nonzero solution to (3.5).

Proof. Let $u$ be the eigenvector of $\mathcal{A}$ associated with the eigenvalue $r(\mathcal{A})$. Take $\delta>0$. Then

$$
R(\delta u)=\mathcal{A}(\delta u)+\int D(x, \tilde{z})\left(\frac{f(\delta u(\tilde{z}))}{1-\tilde{s}+f(\delta u(\tilde{z}))}-\frac{f^{\prime}(0) \delta u(\tilde{z})}{1-\tilde{s}}\right) \sigma(\mathrm{d} \tilde{s}, \mathrm{~d} \tilde{z}) .
$$

Since $f$ is twice differentiable in a neighbourhood of 0 , from assumption (f), and the support of $\sigma$ is restricted to $[0,1-\varepsilon] \times \Omega$ by assumption $(\mathrm{g})$, the second term on the right-hand side of equation (A.3) is $o\left(\delta\|u\|^{2}\right)$ as $\delta \rightarrow 0$. Since $u$ is an eigenvector of $\mathcal{A}$ we have $u \in \stackrel{\circ}{K}$ and $R(\delta u)=r(\mathcal{A}) \delta u+o\left(\delta\|u\|^{2}\right)$. By assumption $r(\mathcal{A})>1$, so, for a sufficiently small $\delta>0$, $\delta u \leq R(\delta u)$. The set of continuous functions bounded below by $\delta u$ and bounded above by 1 form a closed convex set. It follows from the monotonicity of $R$ (part (iii) of Proposition A.1) that $R$ maps this set into itself. Since $R$ is a compact map (part (ii) of Proposition A.1), we can apply the Schauder fixed point theorem (see [14, Theorem 5.1.2]) to conclude that there exists at least one nonzero solution to equation (3.5). To complete the proof of Lemma A.2, we note, from the cone limit set trichotomy, that $R$ can have at most one nonzero fixed point.

Lemmas A.1 and A.2 complete the proof of Theorem 3.2.

\section{A.3. Proof of Theorem 4.1}

First note that the functionals defined by (4.3) and (4.4) are indeed probability generating functionals. Specifically, each of these is the probability generating functional of an MPC, where the offspring from the member of the population located at $(s, z)$ at time $t$ is generated according to an inhomogeneous Poisson process with intensity measure $f^{\prime}(0) D(\cdot, z) \sigma$ and the original member of the population survives to time $t+1$ with probability $s$.

The proof proceeds by induction on $t$. Let $\mathcal{V}_{0}$ denote the set $\left\{h: h \in \mathcal{V}, \inf _{s, z} h(s, z)>0\right\}$. Since $\left\{\mu_{n, 0}\right\}_{n=1}^{\infty}$ converges weakly to some point process $\mu_{0}$, the probability generating functionals $G_{n, 0}[h]$ converge to $G_{0}[h]$ for each continuous $h \in \mathcal{V}_{0}$; see [7, Proposition 11.1.VIII]. Now assume that, for some $T \geq 0, G_{n, T}[h] \rightarrow G_{T}[h]$ for each continuous $h \in \mathcal{V}_{0}$. The probability generating functional of $\mu_{n, T+1}$ is

$$
\begin{aligned}
G_{n, T+1}[h] & =\mathbb{E}\left(\prod_{i=1}^{n}\left(X_{i, T+1}^{n} h\left(s_{i}, z_{i}\right)+1-X_{i, T+1}^{n}\right)\right) \\
& =\mathbb{E}\left(\mathbb{E}\left(\prod_{i=1}^{n}\left(X_{i, T+1}^{n} h\left(s_{i}, z_{i}\right)+1-X_{i, T+1}^{n}\right) \mid X_{T}^{n}, s^{n}, z^{n}\right)\right) .
\end{aligned}
$$


Note that $\mathbb{E}\left(X_{i, T+1}^{n} \mid X_{T}^{n}, s^{n}, z^{n}\right)=U\left(s_{i}\right) X_{i, T}^{n}+f\left(C_{i}(T)\right)\left(1-X_{i, T}^{n}\right)$. Therefore,

$$
\begin{aligned}
G_{n, T+1}[h] & \\
& =\mathbb{E}\left(\prod_{i=1}^{n}\left(X_{i, T}^{n} U\left(s_{i}\right)\left(h\left(s_{i}, z_{i}\right)-1\right)+1+\left(1-X_{i, T}^{n}\right) f\left(C_{i}(t)\right)\left(h\left(s_{i}, z_{i}\right)-1\right)\right)\right) \\
& =\mathbb{E}\left(\prod_{i=1}^{n}\left(1+X_{i, T}^{n} U\left(s_{i}\right)\left(h\left(s_{i}, z_{i}\right)-1\right)\right)\left(1+\left(1-X_{i, T}^{n}\right) f\left(C_{i}(T)\right)\left(h\left(s_{i}, z_{i}\right)-1\right)\right)\right) .
\end{aligned}
$$

Define

$$
\tilde{G}_{n, T+1}[h]:=\mathbb{E}\left(\prod_{i=1}^{n}\left(1+X_{i, T}^{n} U\left(s_{i}\right)\left(h\left(s_{i}, z_{i}\right)-1\right)\right)\left(1+f\left(C_{i}(T)\right)\left(h\left(s_{i}, z_{i}\right)-1\right)\right)\right) .
$$

We want to show that $\left|G_{n, T+1}[h]-\tilde{G}_{n, T+1}[h]\right| \rightarrow 0$ as $n \rightarrow \infty$. We have

$$
\begin{aligned}
\left|G_{n, T+1}[h]-\tilde{G}_{n, T+1}[h]\right| & \\
=\mathbb{E} & \mid\left(\prod_{i=1}^{n}\left(1+X_{i, T}^{n} U\left(s_{i}\right)\left(h\left(s_{i}, z_{i}\right)-1\right)\right)\left(1+\left(1-X_{i, T}^{n}\right) f\left(C_{i}(T)\right)\left(h\left(s_{i}, z_{i}\right)-1\right)\right)\right) \\
& \times\left(1-\prod_{i=1}^{n}\left(1+X_{i, T}^{n} f\left(C_{i}(T)\right)\left(h\left(s_{i}, z_{i}\right)-1\right)\right)\right) \mid \\
\leq \mathbb{E} \mid 1 & -\prod_{i=1}^{n}\left(1+X_{i, T}^{n} f\left(C_{i}(T)\right)\left(h\left(s_{i}, z_{i}\right)-1\right)\right) \mid .
\end{aligned}
$$

Clearly, $\prod_{i=1}^{n}\left(1+X_{i, T}^{n} f\left(C_{i}(T)\right)\left(h\left(s_{i}, z_{i}\right)-1\right)\right) \leq 1$. Let $h_{0}=\inf _{s, z} h(s, z)$. Then

$$
\begin{aligned}
\mathbb{E} \mid 1- & \prod_{i=1}^{n}\left(1+X_{i, T}^{n} f\left(C_{i}(T)\right)\left(h\left(s_{i}, z_{i}\right)-1\right)\right) \mid \\
& \leq 1-\mathbb{E}\left(\exp \left(\sum_{i=1}^{n} \log \left(1-\left(1-h_{0}\right) X_{i, t}^{n} f\left(C_{i}(T)\right)\right)\right)\right) \\
& \leq 1-\mathbb{E}\left(\exp \left(\left(\log h_{0}\right) \sum_{i=1}^{n} X_{i, T}^{n} f\left(C_{i}(T)\right)\right)\right),
\end{aligned}
$$

where the second inequality follows from the bound $\log \left(1-\left(1-h_{0}\right) x\right) \geq\left(\log h_{0}\right) x$ for $x \in[0,1]$. From assumption (f), $f(x) \leq f^{\prime}(0) x$ for $x \in[0, \infty)$. Therefore,

$$
\begin{aligned}
\mathbb{E} \mid 1- & \prod_{i=1}^{n}\left(1+X_{i, T}^{n} f\left(C_{i}(T)\right)\left(h\left(s_{i}, z_{i}\right)-1\right)\right) \mid \\
& \leq 1-\mathbb{E}\left(\exp \left(\left(\log h_{0}\right) f^{\prime}(0) \sum_{i=1}^{n} X_{i, T}^{n} C_{i}(T)\right)\right) \\
& \leq 1-\mathbb{E}\left(\exp \left(\left(\log h_{0}\right) f^{\prime}(0) n^{-1} \bar{D}\left(\sum_{i=1}^{n} X_{i, t}^{n}\right)^{2}\right)\right),
\end{aligned}
$$


where the final inequality comes from assumption (c). Lemma A.3, given at the end of this proof, states that $\exp \left(\alpha \sum_{i=1}^{n} X_{i, t}^{n}\right)$ is uniformly integrable for any $\alpha>0$. Hence, $\left(\sum_{i=1}^{n} X_{i, t}^{n}\right)^{2}$ is uniformly integrable and $n^{-1}\left(\sum_{i=1}^{n} X_{i, t}^{n}\right)^{2} \stackrel{\mathbb{P}}{\rightarrow} 0$. Therefore, the right-hand side of inequality (A.5) goes to zero as required.

To examine the limit of $\tilde{G}_{n, T+1}(h)$, given in equation (A.4), as $n \rightarrow \infty$, we note that

$$
\begin{aligned}
& \sum_{i=1}^{n} \log \left(1+f\left(C_{i}(T)\right)\left(h\left(s_{i}, z_{i}\right)-1\right)\right) \\
& \quad=\sum_{i=1}^{n} f\left(C_{i}(T)\right)\left(h\left(s_{i}, z_{i}\right)-1\right)+\varepsilon_{n, 1} \\
& \quad=\sum_{i=1}^{n} f^{\prime}(0)\left(n^{-1} \sum_{j=1}^{n} X_{j, T}^{n} D\left(z_{i}, z_{j}\right)\right)\left(h\left(s_{i}, z_{i}\right)-1\right)+\varepsilon_{n, 1}+\varepsilon_{n, 2} \\
& \quad=f^{\prime}(0) \sum_{j=1}^{n} X_{j, T}^{n}\left(n^{-1} \sum_{i=1}^{n} D\left(z_{i}, z_{j}\right)\left(h\left(s_{i}, z_{i}\right)-1\right)\right)+\varepsilon_{n, 1}+\varepsilon_{n, 2} \\
& =f^{\prime}(0) \sum_{j=1}^{n} X_{j, T}^{n} \int D\left(z, z_{j}\right)(h(s, z)-1) \sigma_{n}(\mathrm{~d} s, \mathrm{~d} z)+\varepsilon_{n, 1}+\varepsilon_{n, 2},
\end{aligned}
$$

where

$$
\begin{gathered}
\varepsilon_{n, 1}=\sum_{i=1}^{n}\left(\log \left(1+f\left(C_{i}(t)\right)\left(h\left(s_{i}, z_{i}\right)-1\right)\right)-f\left(C_{i}(t)\right)\left(h\left(s_{i}, z_{i}\right)-1\right)\right), \\
\varepsilon_{n, 2}=\sum_{i=1}^{n}\left(f\left(C_{i}(t)\right)\left(h\left(s_{i}, z_{i}\right)-1\right)-f^{\prime}(0) C_{i}(t)\left(h\left(s_{i}, z i\right)-1\right)\right) .
\end{gathered}
$$

Let $\hat{G}_{n, T+1}[h]$ denote

$$
\begin{aligned}
& \mathbb{E}\left(\left(\prod_{i=1}^{n}\left(1+X_{i, T}^{n} U\left(s_{i}\right)\left(h\left(s_{i}, z_{i}\right)-1\right)\right)\right)\right. \\
& \left.\quad \times \exp \left(f^{\prime}(0) \sum_{j=1}^{n} X_{j, t}^{n} \int D\left(z, z_{j}\right)(h(s, z)-1) \sigma_{n}(\mathrm{~d} s, \mathrm{~d} z)\right)\right) .
\end{aligned}
$$

To show that $\left|\tilde{G}_{n, T+1}[h]-\hat{G}_{n, T+1}[h]\right| \rightarrow 0$ as $n \rightarrow \infty$ note that the difference is bounded by

$$
\begin{aligned}
& \left|\tilde{G}_{n, T+1}[h]-\hat{G}_{n, T+1}[h]\right| \\
& \leq \mathbb{E}\left(\left(\prod_{i=1}^{n}\left(1+X_{i, T}^{n} U\left(s_{i}\right)\left(h\left(s_{i}, z_{i}\right)-1\right)\right)\right)\right. \\
& \left.\quad \quad \times \exp \left(f^{\prime}(0) \sum_{j=1}^{n} X_{j, T}^{n} \int D\left(z, z_{j}\right)(h(s, z)-1) \sigma_{n}(\mathrm{~d} s, \mathrm{~d} z)\right)\left|\left(\exp \left(\varepsilon_{n, 1}+\varepsilon_{n, 2}\right)-1\right)\right|\right) \\
& \leq \mathbb{E}\left|\left(\exp \left(\varepsilon_{n, 1}+\varepsilon_{n, 2}\right)-1\right)\right| \\
& \leq \mathbb{E} \exp \left(\left|\varepsilon_{n, 1}\right|+\left|\varepsilon_{n, 1}\right|\right)-1 .
\end{aligned}
$$


Note that $|\log (1-x)+x| \leq-\log \left(h_{0}\right) /\left(1-h_{0}\right)^{2} x^{2}$ for $x \in\left[0,1-h_{0}\right]$. From assumptions (c) and (f), $\left|\varepsilon_{n, 1}\right|$ is bounded,

$$
\left|\varepsilon_{n, 1}\right| \leq \frac{-\log \left(h_{0}\right)}{\left(1-h_{0}\right)^{2}} \sum_{i=1}^{n} f^{2}\left(C_{i}(t)\right) \leq \frac{-\log \left(h_{0}\right)}{\left(1-h_{0}\right)^{2}}\left(f^{\prime}(0) \bar{D}\right)^{2} n^{-1}\left(\sum_{i=1}^{n} X_{i, t}\right)^{2} .
$$

From assumption (f), there exists a $c_{1}>0$ such that $\left|f(x)-f^{\prime}(0) x\right| \leq c_{1} x^{2}$ for $x \in[0, \infty)$. Therefore, $\varepsilon_{n, 2}$ can also be bounded,

$$
\left|\varepsilon_{n, 2}\right| \leq \sum_{i=1}^{n}\left|f\left(C_{i}(T)\right)-f^{\prime}(0) C_{i}(T)\right| \leq c_{2} n^{-1}\left(\sum_{i=1}^{n} X_{i, T}^{n}\right)^{2}
$$

for some $c_{2}>0$. Hence,

$$
\left|\varepsilon_{n, 1}\right|+\left|\varepsilon_{n, 2}\right|<c_{3} n^{-1}\left(\sum_{j=1}^{n} X_{j, T}^{n}\right)^{2}
$$

for some $c_{3}>0$. Since $\exp \left(\alpha \sum_{i=1}^{n} X_{i, T}^{n}\right)$ is uniformly integrable (Lemma A.3) for any $\alpha>0$ and $n^{-1}\left(\sum_{i=1}^{n} X_{i, T}^{n}\right)^{2} \stackrel{\mathbb{P}}{\rightarrow} 0$, the right-hand side of inequality (A.6) goes to 0 .

We may now express $G_{n, T}\left[G_{1}[h \mid(s, z)]\right]$ as

$$
\begin{aligned}
G_{n, T}\left[G_{1}[h \mid(s, z)]\right]=\mathbb{E}( & \left(\prod_{i=1}^{n}\left(X_{i, T}^{n}\left(1-U\left(s_{i}\right)\left(1-h\left(s_{i}, z_{i}\right)\right)\right)+1-X_{i, T}^{n}\right)\right) \\
& \left.\times \exp \left(f^{\prime}(0) \sum_{j=1}^{n} X_{j, T}^{n} \int D\left(z, z_{j}\right)(h(s, z)-1) \sigma(\mathrm{d} s, \mathrm{~d} z)\right)\right) .
\end{aligned}
$$

We now show that $\left|\hat{G}_{n, T+1}[h]-G_{n, T}\left[G_{1}[h \mid(s, z)]\right]\right| \rightarrow 0$ as $n \rightarrow \infty$. Let $g\left(h ; X_{T}^{n}, \sigma_{n}\right)$ denote

$$
\sum_{j=1}^{n} X_{j, T}^{n}\left(\int D\left(z, z_{j}\right)(h(s, z)-1) \sigma_{n}(\mathrm{~d} s, \mathrm{~d} z)-\int D\left(z, z_{j}\right)(h(s, z)-1) \sigma(\mathrm{d} s, \mathrm{~d} z)\right) .
$$

Noting that $1+X_{i, T} U\left(s_{i}\right)\left(h\left(s_{i}, z_{i}\right)-1\right)=X_{i, T}\left(1-U\left(s_{i}\right)\left(1-h\left(s_{i}, z_{i}\right)\right)\right)+1-X_{i, T}$, we see that

$$
\begin{aligned}
& \mid \hat{G}_{n, T+1}[h]- G_{n, T}\left[G_{1}[h \mid(s, z)]\right] \mid \\
&=\mid \mathbb{E}(\left(\prod_{i=1}^{n}\left(X_{i, T}^{n}\left(1-U\left(s_{i}\right)\left(1-h\left(s_{i}, z_{i}\right)\right)\right)+1-X_{i, T}^{n}\right)\right) \\
& \times \exp \left(f^{\prime}(0) \sum_{j=1}^{n} X_{j, T}^{n} \int D\left(z, z_{j}\right)(h(s, z)-1) \sigma(\mathrm{d} s, \mathrm{~d} z)\right) \\
&\left.\times\left(\exp \left(g\left(h ; X_{T}^{n}, \sigma_{n}\right)\right)-1\right)\right) \mid \\
& \leq \mathbb{E}\left|\exp \left(g\left(h ; X_{T}^{n}, \sigma_{n}\right)\right)-1\right|
\end{aligned}
$$


From assumption (c) we have $\left|\mathscr{g}\left(h ; X_{T}^{n}, \sigma_{n}\right)\right| \leq 2 \bar{D} \sum_{j=1}^{n} X_{j, T}$. Therefore, $\exp \left(\mathscr{g}\left(h, X_{T}^{n}, \sigma_{n}\right)\right)$ is uniformly integrable. To show that the right-hand side of (A.7) converges to 0 as $n \rightarrow \infty$, it is sufficient to show that $g\left(h ; X_{T}^{n}, \sigma_{n}\right)$ converges to 0 in probability. Define the measures $\sigma_{n}^{(h)}$ and $\sigma^{(h)}$ as being the measures with Radon-Nikodym derivative $(1-h(s, z))$ with respect to $\sigma_{n}$ and $\sigma$, respectively. Since $h$ is a continuous function in $\mathcal{V}_{0}$ and, from assumption (e), $\sigma_{n} \stackrel{\mathrm{D}}{\rightarrow} \sigma$, it follows that $\sigma_{n}^{(h)} \stackrel{\mathrm{D}}{\rightarrow} \sigma^{(h)}$. By assumption (c), the kernel $D(\tilde{z}, z)$ defines a uniformly bounded and equicontinuous family of functions on $\Omega$. Therefore, we can apply a small modification of [27, Theorem 3.1] to show that

$$
\sup _{z \in \Omega}\left(\int D(\tilde{z}, z)(h(\tilde{s}, \tilde{z})-1) \sigma_{n}(\mathrm{~d} \tilde{s}, \mathrm{~d} \tilde{z})-\int D(\tilde{z}, z)(h(\tilde{s}, \tilde{z})-1) \sigma(\mathrm{d} \tilde{s}, \mathrm{~d} \tilde{z})\right) \stackrel{\mathbb{P}}{\rightarrow} 0 .
$$

Since $\exp \left(\alpha \sum_{i=1}^{n} X_{i, t}^{n}\right)$ is uniformly integrable for any $\alpha>0$ (Lemma A.3), so is $\sum_{i=1}^{n} X_{i, t}^{n}$. Therefore, $g\left(h ; X^{n}, \sigma_{n}\right) \stackrel{\mathbb{P}}{\rightarrow} 0$. Hence, $\left|\hat{G}_{n, T+1}[h]-G_{n, T}\left[G_{1}[h \mid(s, z)]\right]\right| \rightarrow 0$.

It is easily checked that if $h$ is a continuous function in $\mathcal{V}_{0}$ then $G_{1}[h \mid(s, z)]$ is also a continuous function in $\mathcal{V}_{0}$. Applying the inductive hypothesis, we see that $G_{n, T}\left[G_{1}[h \mid(s, z)]\right] \rightarrow$ $G_{T}\left[G_{1}[h \mid(s, z)]\right]$ for all continuous $h \in \mathcal{V}_{0}$. Hence, $G_{n, T+1}[h] \rightarrow G_{T}\left[G_{1}[h \mid(s, z)]\right]=$ $G_{T+1}[h]$ for all continuous $h \in \mathcal{V}_{0}$. This completes the proof of Theorem 4.1.

Lemma A.3. Suppose that, for all $\alpha>0, \sup _{n} \mathbb{E}\left(\exp \left(\alpha \sum_{i=1}^{n} X_{i, 0}^{n}\right)\right)<\infty$. Then, for all $\alpha>0$ and all $t \geq 0, \sup _{n} \mathbb{E}\left(\exp \left(\alpha \sum_{i=1}^{n} X_{i, t}^{n}\right)\right)<\infty$.

Proof. The proof proceeds by induction on $t$. By assumption, $\sup _{n} \mathbb{E}\left(\exp \left(\alpha \sum_{i=1}^{n} X_{i, 0}^{n}\right)\right)<$ $\infty$ for all $\alpha>0$. Suppose now that, for some $T \geq 0, \sup _{n} \mathbb{E}\left(\exp \left(\alpha \sum_{i=1}^{n} X_{i, T}^{n}\right)\right)<\infty$ for all $\alpha>0$. Then

$$
\begin{aligned}
\mathbb{E}\left(\exp \left(\alpha \sum_{i=1}^{n} X_{i, T+1}^{n}\right)\right) \\
=\mathbb{E}\left(\mathbb{E}\left(\exp \left(\alpha \sum_{i=1}^{n} X_{i, T+1}^{n}\right) \mid X_{T}^{n}, s^{n}, z^{n}\right)\right) \\
=\mathbb{E}\left(\prod _ { i = 1 } ^ { n } \left(\left(1-s_{i}+s_{i} \exp (\alpha)\right) X_{i, T}^{n}\right.\right. \\
\left.\left.\quad+\left(1-f\left(C_{i}(T)\right)+f\left(C_{i}(T)\right) \exp (\alpha)\right)\left(1-X_{i, T}^{n}\right)\right)\right) \\
\leq \mathbb{E}\left(\prod_{i=1}^{n}\left(X_{i, T}^{n} \exp (\alpha)+1+f\left(C_{i}(T)\right)(\exp (\alpha)-1)\right)\right) \\
\leq \mathbb{E}\left(\exp \left(\sum_{i=1}^{n}\left(\exp (\alpha) X_{i, T}^{n}+f\left(C_{i}(T)\right)(\exp (\alpha)-1)\right)\right)\right) \\
\quad \leq \mathbb{E}\left(\exp \left(\sum_{i=1}^{n}\left(\exp (\alpha)\left(1+f^{\prime}(0) \bar{D}\right) \sum_{i=1}^{n} X_{i, T}^{n}\right)\right) .\right.
\end{aligned}
$$


Since $\sup _{n} \mathbb{E}\left(\exp \left(\alpha \sum_{i=1}^{n} X_{i, T}^{n}\right)\right)<\infty$ for all $\alpha>0$, inequality (A.8) implies that, for all $\alpha>0, \sup _{n} \mathbb{E}\left(\exp \left(\alpha \sum_{i=1}^{n} X_{i, T+1}^{n}\right)\right)<\infty$.

\section{A.4. Proof of Theorem 4.2}

Let $K$ denote the cone of continuous positive functions on $\Omega$ and let $K_{1}=\{\phi: \phi \in K$, $\phi \leq 1\}$. Define the operator

$$
R \phi(z)=\exp \left(-f^{\prime}(0) \int D(\tilde{z}, z)\left(\frac{1-\phi(\tilde{z})}{1-U(\tilde{s}) \phi(\tilde{z})}\right) \sigma(\mathrm{d} \tilde{s}, \mathrm{~d} \tilde{z})\right), \quad \phi \in K_{1} .
$$

Note that, under assumption $(\mathrm{g}), 1-U(s) \phi(z)>0$ on the support of $\sigma$ for any $\phi \in K_{1}$ and, therefore, $R: K_{1} \mapsto K_{1}$. From [22, Theorem 3.1], the asymptotic extinction probability of a multiplicative population chain starting at $(s, z)$ is given by the smallest nonnegative solution to $h(s, z)=G_{1}[h \mid(s, z)]$. Denote this solution by $h^{*}(s, z)$, and define

$$
\psi[h](z)=\exp \left(-f^{\prime}(0) \int D(\tilde{z}, z)(1-h(\tilde{s}, \tilde{z})) \sigma(\mathrm{d} \tilde{s}, \mathrm{~d} \tilde{z})\right) .
$$

Note that, for any $h \in K_{1}, \psi[h] \in K_{1}$. Substituting (A.9) into $G_{1}[h \mid(s, z)]$, we obtain

$$
h^{*}(s, z)=\left(1-U(s)+U(s) h^{*}(s, z)\right) \psi\left[h^{*}\right](z) .
$$

Hence, $h^{*}$ satisfies

$$
h^{*}(s, z)=\frac{(1-U(s)) \psi\left[h^{*}\right](z)}{1-U(s) \psi\left[h^{*}\right](z)}
$$

on the support of $\sigma$. Substituting this back into (A.9), we see that $\psi\left[h^{*}\right](z)$ satisfies the equation $\psi(z)=R \psi(z)$. From the definition of $R, \psi(z)=1$ for some $z \in \Omega$ if and only if $h=1, \sigma$-almost everywhere. Since the right-hand side of (A.10) is increasing in $\psi, h^{*}$ will be determined by the smallest nonnegative fixed point of the operator $R$.

Recall that $1-\exp (-x) \leq x$ for all $x \geq 0$ with equality if and only if $x=0$. Therefore,

$$
\begin{aligned}
1-R \psi(z) & \leq f^{\prime}(0) \int D(\tilde{z}, z)\left(\frac{1-\psi(\tilde{z})}{1-U(\tilde{s}) \psi(\tilde{z})}\right) \sigma(\mathrm{d} \tilde{s}, \mathrm{~d} \tilde{z}) \\
& \leq f^{\prime}(0) \int \frac{D(\tilde{z}, z)}{(1-U(\tilde{s}))}(1-\psi(\tilde{z})) \sigma(\mathrm{d} \tilde{s}, \mathrm{~d} \tilde{z}) \\
& =\mathcal{A}(1-\psi)(z),
\end{aligned}
$$

where the equality follows since $D$ is symmetric with respect to its arguments from assumption (c). Note that we have equality if and only if $\psi(z)=1$ for all $z$. As in the proof of Theorem 3.2, the operator $\mathcal{A}$ is strongly positive on $K$. If $\psi^{*}$ is the smallest nonnegative fixed point of $R$ then

$$
1-\psi^{*}=1-R \psi^{*} \leq \mathcal{A}\left(1-\psi^{*}\right) .
$$

Let $r(\mathcal{A})$ denote the spectral radius of $\mathcal{A}$. Since $\psi^{*} \in K_{1}, 1-\psi^{*} \in K$ and we can apply Corollary A.1 to conclude that if $r(\mathcal{A})<1$ then $\psi^{*}(z)=1$ for all $z \in \Omega$. Furthermore, if $r(\mathcal{A})=1$ then $1-\psi^{*}=\mathcal{A}\left(1-\psi^{*}\right)$. Since inequality (A.11) is strict for $\psi^{*} \neq 1$, it follows that $\psi^{*}(z)=1$ for all $z \in \Omega$. Hence, if $r(\mathcal{A}) \leq 1$ then $h^{*}(s, z)=1$ on the support of $\sigma$.

We now consider the case of $r(A)>1$. We aim to find a convex subset of $K_{1}$, not containing the function 1, such that $R$ maps this set to itself. We can then apply the Schauder fixed point 
theorem [14, Theorem 5.1.2] to show that $\psi^{*} \neq 1$. Clearly $R 0 \in K_{1}$. Since the function $(1-x)(1-s x)^{-1}$ is decreasing in $x>0$ for any $s \in[0,1-\varepsilon]$, it follows that, for any $\phi_{1}, \phi_{2} \in K_{1}$ such that $\phi_{1} \leq \phi_{2}$, we have $R \phi_{1} \leq R \phi_{2}$. From the Krein-Rutman theorem (see Theorem A.1), $r(\mathcal{A})$ is an eigenvalue of $\mathcal{A}$ and has an associated eigenvector $u \in K$. For any $\delta>0$ sufficiently small, we have $R(1-\delta u)=R(1)-\delta \mathcal{A} u+\varepsilon_{\delta, u}=1-\delta r(A) u+\varepsilon_{\delta, u}$, where

$$
\begin{aligned}
\varepsilon_{\delta, u}= & R(1-\delta u)-1+f^{\prime}(0) \int D(\tilde{z}, z) \frac{\delta u(\tilde{z})}{1-\tilde{s}+\tilde{s} \delta u(\tilde{z})} \sigma(\mathrm{d} \tilde{s}, \mathrm{~d} \tilde{z}) \\
& +\delta \int D(\tilde{z}, z) u(\tilde{z})\left(\frac{1}{1-\tilde{s}+\delta \tilde{s} u(\tilde{z})}-\frac{1}{1-\tilde{s}}\right) \sigma(\mathrm{d} \tilde{s}, \mathrm{~d} \tilde{z}) .
\end{aligned}
$$

It can be seen that $\varepsilon_{\delta, u}$ is $O\left(\delta^{2}\right)$ since

$$
\begin{aligned}
\mid R(1 & -\delta u)-1+f^{\prime}(0) \int D(\tilde{z}, z) \frac{\delta u(\tilde{z})}{1-\tilde{s}+\tilde{s} \delta u(\tilde{z})} \sigma(\mathrm{d} \tilde{s}, \mathrm{~d} \tilde{z}) \mid \\
& \leq\left(f^{\prime}(0)\right)^{2}\left(\int D(\tilde{z}, z) \frac{\delta u(\tilde{z})}{1-\tilde{s}+\tilde{s} \delta u(\tilde{z})} \sigma(\mathrm{d} \tilde{s}, \mathrm{~d} \tilde{z})\right)^{2} \\
& \leq\left(f^{\prime}(0)\right)^{2} \bar{D}^{2} \delta^{2}\left(\int \frac{u(\tilde{z})}{1-\tilde{s}} \sigma(\mathrm{d} \tilde{s}, \mathrm{~d} \tilde{z})\right)^{2}
\end{aligned}
$$

and

$$
\int D(\tilde{z}, z) u(\tilde{z})\left|\frac{1}{1-\tilde{s}+\delta \tilde{s} u(\tilde{z})}-\frac{1}{1-\tilde{s}}\right| \sigma(\mathrm{d} \tilde{s}, \mathrm{~d} \tilde{z}) \leq \delta \bar{D} \int \frac{u(\tilde{z})}{(1-\tilde{s})^{2}} \sigma(\mathrm{d} \tilde{s}, \mathrm{~d} \tilde{z}),
$$

where both integrals are finite from assumption $(\mathrm{g})$. Therefore, for $\delta>0$ sufficiently small, $R(1-\delta u) \leq 1-\delta u$ when $r(\mathcal{A})>1$. Take the convex set of functions

$$
K_{u}:=\{\phi: \phi \in K, \phi \leq 1-\delta u\} .
$$

We see that $R: K_{u} \mapsto K_{u}$ and hence, from the Schauder fixed point theorem, there exists $\psi^{*} \in K_{u}$ satisfying $\psi=R \psi$ if $r(\mathcal{A})>1$. Hence, if $r(\mathcal{A})>1$ then $h^{*}(s, z)<1$ for all $(s, z)$ in the support of $\sigma$. Finally, $h^{*}(s, z)$ gives the asymptotic extinction probability of a multiplicative population chain starting with a single point at $(s, z)$. Since the chains starting from two different points are conditionally independent, the asymptotic probability of extinction for the limiting metapopulation is $\mathbb{E}\left(\prod_{i} h^{*}\left(s_{i}, z_{i}\right)\right)=G_{0}\left[h^{*}\right]$, where the product is over the occupied patches in the limiting metapopulation at $t=0$. This completes the proof of Theorem 4.2.

\section{Acknowledgements}

We are grateful to the referee for helpful comments and suggestions. This work is supported by the Australian Research Council (Discovery grant DP120102398 and Centre of Excellence for Mathematics and Statistics of Complex Systems).

\section{References}

[1] Ball, F. (1983). The threshold behaviour of epidemic models. J. Appl. Prob. 20, 227-241.

[2] Ball, F. AND Clancy, D. (1993). The final size and severity of a generalised stochastic multitype epidemic model. J. Appl. Prob. 25, 721-736.

[3] Ball, F. and Donnelly, P. (1995). Strong approximations for epidemic models. Stoch. Proc. Appl. 55, 1-21.

[4] Barbour, A. D. (2010). Coupling a branching process to an infinite dimensional epidemic process. ESAIM Prob. Statist. 14, 53-64. 
[5] Buckley, F. M. And Pollett, P. K. (2010). Limit theorems for discrete-time metapopulation models. Prob. Surveys, 7, 53-83.

[6] Courchamp, F., Berec, L. and Gascoigne, J. (2008). Allee Effects in Ecology and Conservation. Oxford University Press, New York.

[7] Daley, D. J. and Vere-Jones, D. (2008). An Introduction to the Theory of Point Processes, Vol. 2, Springer, New York.

[8] Dautray, R. and Lions, J.-L. (1990). Mathematical Analysis and Numerical Methods for Science and Technology, Vol. 3. Springer, Berlin.

[9] Durrett, R. (1999). Stochastic spatial models. SIAM Rev. 41, 677-718.

[10] Hanski, I. (1994). A practical model of metapopulation dynamics. J. Anim. Ecol. 63, 151-162.

[11] Hanski, I., Alho, J. and Moilanen, A. (2000). Estimating the parameters of survival and migration of individuals in metapopulations. Ecology 81, 239-251.

[12] Hanski, I. and Ovaskainen, O. (2003). Metapopulation theory for fragmented landscapes. Theoret. Pop. Biol. 64, 119-127.

[13] Hirsch, M. W. And Smith, H. (2005). Monotone maps: a review. J. Difference Equat. 11, 379-398.

[14] Istratescu, V. I. (1981). Fixed Point Theory: An Introduction. D. Reidel, Dordrecht.

[15] Kallenberg, O. (2002). Foundations of Modern Probability, 2nd edn. Springer, New York.

[16] Levins, R. (1969). Some demographic and genetic consequences of environmental heterogeneity for biological control. Bull. Entomol. Soc. Amer. 15, 237-240.

[17] MacCluer, B. D. (2009). Elementary Functional Analysis, Springer, New York.

[18] McVinish, R. And Pollett, P. K. (2010). Limits of large metapopulations with patch-dependent extinction probabilities. Adv. Appl. Prob. 42, 1172-1186.

[19] McVinish, R. ANd Pollett, P. K. (2012). The limiting behaviour of a mainland-island metapopulation. J. Math. Biol. 64, 775-801.

[20] McVinish, R. And Pollett, P. K. (2013). The limiting behaviour of a stochastic patch occupancy model. J. Math. Biol. 67, 693-716.

[21] Mollanen, A. (2004). SPOMSIM: software for stochastic patch occupancy models of metapopulation dynamics. Ecol. Model. 179, 533-550.

[22] Moyal, J. E. (1962). Multiplicative population chains. Proc. R. Soc. London A 266, 518-526.

[23] Ovaskainen, O. and Cornell, S. J. (2006). Asymptotically exact analysis of stochastic metapopulation dynamics with explicit spatial structure. Theoret. Pop. Biol. 69, 13-33.

[24] Ovaskainen, O. AND HANSKI, I. (2001). Spatially structured metapopulation models: global and local assessment of metapopulation capacity. Theoret. Pop. Biol. 60, 281-302.

[25] Penrose, M. D. (2008). Existence and spatial limit theorems for lattice and continuum particle systems. Prob. Surveys, 5, 1-36.

[26] Petit, S., Moilanen, A., Hanski, I. And Baguette, M. (2001). Metapopulation dynamics of the bog fritillary butterfly: movements between habitat patches. Oikos 92, 491-500.

[27] Ranga RaO, R. (1962). Relations between weak and uniform convergence of measures with applications. Ann. Math. Statist. 33, 659-680. 